\title{
Origami MEMS and NEMS
}

\author{
John Rogers, Yonggang Huang, Oliver G. Schmidt, and \\ David H. Gracias
}

\begin{abstract}
In a manner reminiscent of macroscale bending and folding techniques such as origami, the out-of-plane assembly of lithographically micro- and nanopatterned thin films, can be used to fabricate three-dimensional (3D) micro- and nanostructured devices. These 3D devices, including microelectronic circuits, sensors, antennas, metamaterials, robotic, and biomimetic constructs, enable new functionalities and are challenging to fabricate by other methods. In this article, we summarize important features of this set of techniques and the devices assembled thereof, with a focus on functional constructs that have been formed by bending, folding, or buckling. At small size scales, manipulation using manual or even wired probes face daunting practical challenges in terms of cost, scalability, and high-throughput manufacturability; hence we emphasize techniques that manipulate strain in thin films so that they can spontaneously assemble into programmed 3D geometries without the need for any wires or probes.
\end{abstract}

\section{Introduction}

Origami, the ancient art of paper folding that is now practiced in many cultures, broadly refers to a set of techniques to transform planar sheets into three-dimensional (3D) structures. Variants of traditional origami include kirigami, which involves cutting and folding, and pop-up art, which involves gluing, cutting, and folding. Over the last two decades, this comprehensive set of techniques has inspired the transformation of patterned thin films composed of materials other than paper into 3D functional microelectromechanical (MEMS) and nanoelectromechanical systems (NEMS).

There are a number of advantages in this approach especially relevant to the science and engineering of small functional devices. First, the assembly paradigm is compatible with very well-established inherently planar lithographic techniques such as photo, e-beam, and nanoimprint lithography. Hence, in principle, any two-dimensional (2D) pattern, including those with nanometer-sized, high-fidelity features, can be popped up into a 3D structure. Second, these assembly processes can be applied to a wide range of materials ranging from devicegrade single-crystal silicon to cells and biomaterials. Further, folding can be performed in a hands-free and wire-free manner enabling high-throughput and parallel assembly, which is important from a manufacturing perspective. Additionally, a number of adhesive contacts, mechanical latches, and fluidic joints have been developed to self-seal seams after 3D assembly, and consequently, rigid folded structures can be reliably fabricated.

Finally, theoretical advances in mechanics, geometry, origami mathematics, and biomimetics have uncovered design rules for the high-yield self-assembly of complex 3D structures by folding. Consequently, origami assembly approaches have moved from intellectual curiosities into the mainstream of micro- and nanoscale science and engineering. In contrast to previous reviews or broad relevance to this area of origami fabrication and assembly, ${ }^{1-9}$ in this article, we focus on recent advances in functional origami MEMS and NEMS devices.

\section{Folding versus self-folding}

Techniques used to achieve macroscale folding by hand, tweezers, or machines can be challenging to implement at small size scales. Nevertheless, manual folding and popping up of microfabricated metals, polysilicon, and printed ink patterns have been pushed to the limit to realize assembly of complex 3D structures such as towers, ceramic birds, supercapacitors, and lightweight materials (see Figure 1a-d). ${ }^{10-16}$ Sequential folding has been achieved by attaching electrically actuated

John Rogers, Department of Materials Science and Engineering, Frederick Seitz Materials Research Laboratory, University of Illinois at Urbana-Champaign, USA; jrogers@illinois.edu Yonggang Huang, Department of Civil and Environmental Engineering, Department of Mechanical Engineering, Northwestern University, USA; y-huang@northwestern.edu

Oliver G. Schmidt, Institute for Integrative Nanosciences, Leibniz Institute for Solid State and Materials Research Dresden, Material Systems for Nanoelectronics, Chemnitz University of Technology, Germany; 0.schmidt@ifw-dresden.de

David H. Gracias, Department of Chemical and Biomolecular Engineering, Department of Materials Science and Engineering, Johns Hopkins University, USA; dgracias@jhu.edu

DOI: $10.1557 / \mathrm{mrs} .2016 .2$ 

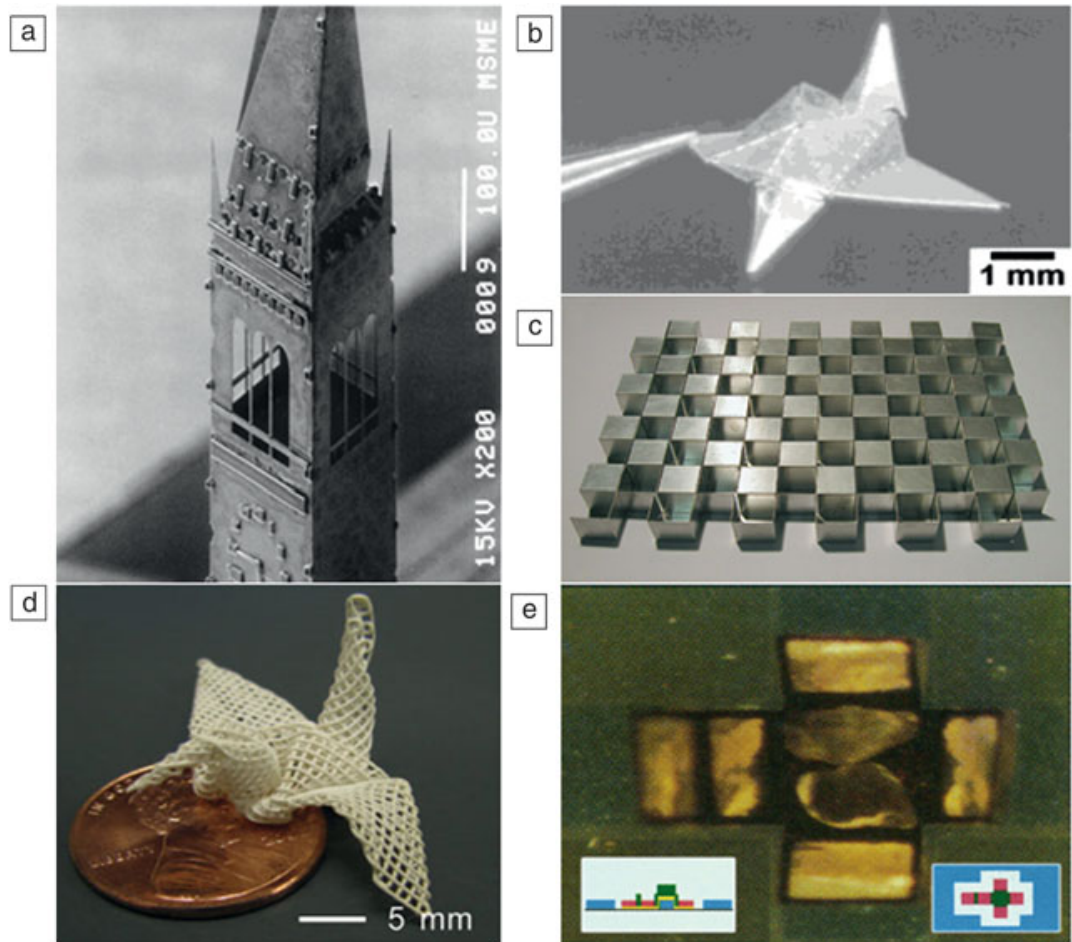

Figure 1. Folded micro-origami. (a) A high-resolution image of a pop-up silicon tower, which was assembled using a probe on a manual manipulator. Image courtesy of Elliot Hui. White scale bar indicates $100 \mu \mathrm{m}$. Reprinted with permission from Reference 11. () 2000 IEEE. (b) Photograph of manually folded and electroplated tessellated silver bird with the tips of tweezers shown at the left. Reprinted with permission from Reference 12. ( 2001 American Chemical Society. (c) Photograph of a lightweight material formed by folding and welding stamped-out patterns of sheet metal. The side of the square element is 25 $\mathrm{mm}$ and the length of the entire cubic core material is $30 \mathrm{~cm}$. Image courtesy of Taketoshi Nojima. Reprinted with permission from Reference 14. () 2007 Nature Publishing Group. (d) Optical image of a titanium oxide crane produced by manual folding of a printed pattern followed by annealing. Reprinted with permission from Reference 15. () 2010 Wiley. (e) Wired electrochemically actuated folding of a $300-\mu \mathrm{m}$ box using doped polypyrrole (PPy)-gold bilayer hinges and benzocyclobenzene (BCB) rigid segments. The insets depict top and side views of different micropatterned layers, including PPy (green), gold (yellow), BCB (red), and chromium (blue), which are used to attach the central panel to the substrate. Reprinted with permission from Reference 18. (c) 1995 American Association for the Advancement of Science.

wired hinges such as those composed of polypyrrole (Figure 1e) or shape-memory actuators. ${ }^{17-19}$ However, popping up structures by hand or even wiring individual hinges to achieve folding can restrict manufacturing throughput, miniaturization, and off-chip assembly.

Parallel assembly of small 3D devices by origami approaches necessitates manipulation of thin films using forces derived from material properties such as strain mismatch, ${ }^{20-23}$ capillary forces, ${ }^{24-27}$ magnetic forces, ${ }^{28,29}$ shrinking, or swelling. ${ }^{7,30-36}$ Here, the final 3D configuration typically represents a state of equilibrium derived from the minimization of the total system energy comprising the strain energy (in terms of bending energy and membrane energy incorporated in the various stresses within the thin films) of the structures as well as the work done by the external force (e.g., capillary or magnetic forces). Depending on the form of the driving force, various deformation modes (e.g., global buckling and localized wrinkling) and their combinations could occur, thereby enabling the formation of highly complex 3D geometries (see examples shown in Figure 2a-h). For example, with self-actuating materials capable of programmable shape changes, a wide range of 3D surfaces (e.g., spherical caps and saddles with constant Gaussian curvature, and Enneper's surfaces, which are minimal surfaces with zero-mean curvature) can be formed, ${ }^{31,33}$ simply through the use of different patterns of shrinkage and swelling. For thin-sheet configurations, the bending stiffness scales with the cube of thickness, while the tensile stiffness scales linearly with the thickness. Therefore, bending is less costly than compression or tension as thickness decreases, making out-of-plane deformations easy to trigger in thin sheets that are locally growing or shrinking. For 3D structures made of brittle semiconductors or plastic metals, it is also important to control the geometry of the thin sheet as well as the fabrication parameters, such that the strain level in the material can be maintained at a safe level to avoid fracture or delamination.

Another challenge in self-folding, especially of complex geometries, is to solve the inverse problem of uncovering which flat patterns can be programmed to fold up spontaneously into the desired final 3D shape using the forces described previously. In the absence of any wiring or control over local folding pathways, the starting pattern must be optimized to reach a stable and predictable global structure. Significant advances in mechanical modeling, geometric design, and computational origami have provided new insights into this problem. For example, studies on the design of self-folding polyhedra using capillary forces show that compact flat designs (also called nets) yield larger numbers of defect-free polyhedra as compared to more open nets (Figure $2 \mathrm{i}-\mathrm{j}$ ). ${ }^{37}$ Appropriate software and theoretical insights from origami mathematics have begun to allow one to solve inverse origami problems and design significantly complex origami. ${ }^{38-41}$ Other approaches such as four-dimensional printing have been proposed; these methods utilize both planar and nonplanar printed patterns that can change shape after printing. ${ }^{42}$ By utilizing nonplanar starting fold patterns, more complicated geometries could conceivably be formed using 3D printed patterns, albeit with serial and limited linewidth resolution as compared to that which can be generated with planar lithography. 


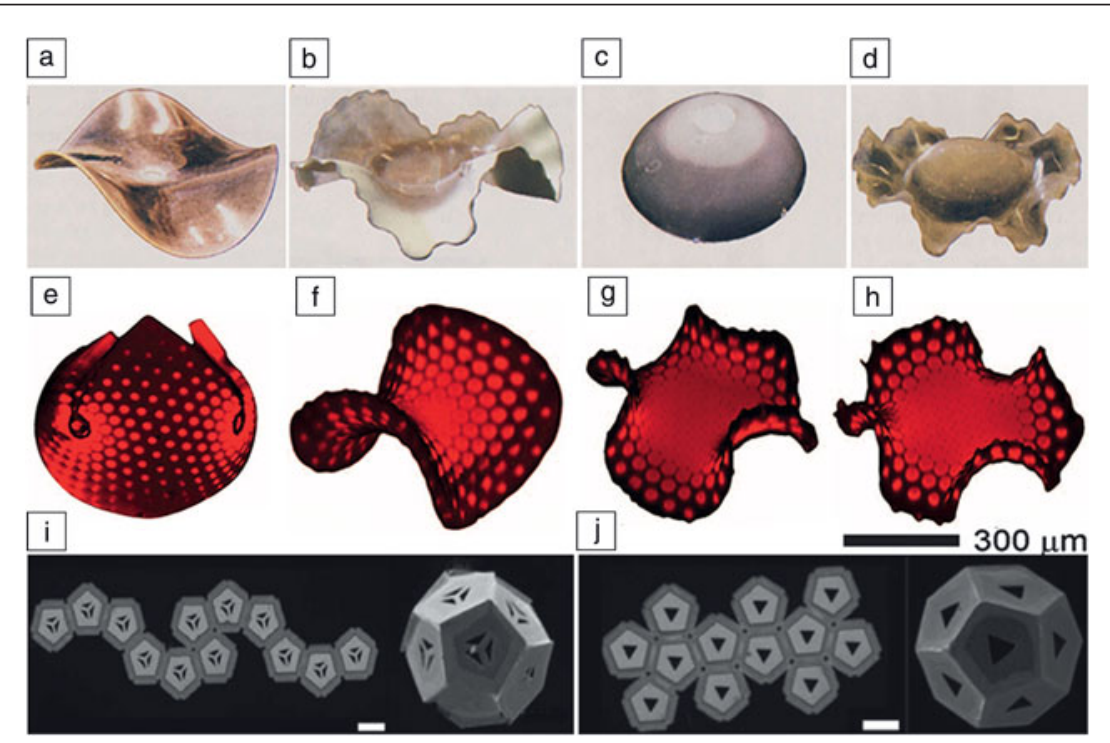

Figure 2. Mechanics and design of self-folded structures. (a-h) 3D structures constructed with $\mathrm{cm}$-scale gel sheets that undergo nonuniform shrinkage. (a) A thick $(t=0.75 \mathrm{~mm})$ sheet that adopts a configuration with only three waves. (b) Thinner $(t=0.3 \mathrm{~mm})$ sheets with two generations of waves. (c) A spherical cap that can be combined with negative curvature margins to obtain a wavy sombrero-like structure (d). (e) A nearly closed spherical shape. (f) A piece of saddle surface. (g-h) Enneper's surfaces (minimal surfaces with zeromean curvature) with three and four nodes. (a-d) Adapted with permission from Reference 31. (C) 2007 American Association for the Advancement of Science. (e-h) Adapted with permission from Reference 33. (C) 2012 American Association for the Advancement of Science. (i-j) Scanning electron micrographs of self-folding metallic dodecahedra (right) which were assembled from planar nets (left) with two and six vertex connections, respectively. The compact net in panel (j) assembles without defects, while the open net in panel (i) assembles into a dodecahedron with misaligned edges. Scale bar shown near the open net and length of the side of each pentagon is $300 \mu \mathrm{m}$. Reprinted with permission from Reference 37. (C) 2011 National Academy of Sciences.

\section{Functional devices}

While origami, kirigami, and pop-up approaches may appear to be art forms, techniques for folding, curving, bending, twisting, and bonding materials have been refined and adapted in engineering to provide true functional value for 3D MEMS and NEMS. In what follows, we review functional micro- and nanoscale devices fabricated using these approaches.

\section{Electrical, electromagnetic, and energy devices}

Complex, 3D micro-/nanostructures are ubiquitous in biology, where they provide versatile functionality in mechanical actuation (cytoskeletons), fluid transport (vascular networks), electrical communication (neural circuits), and others. Analogs do not exist even in the most advanced electronics technologies due to an absence of capabilities for 3D micro-/nanofabrication with high-performance electronic materials such as inorganic semiconductors. For example, techniques such as 3D printing or two-photon polymerization do not permit structuring of device-grade silicon. In contrast, capillary or residual stresses can be used to induce bending in thin, released layers from semiconductor wafers in a way that is naturally compatible with modern planar technologies. The result provides access to $3 \mathrm{D}$ geometries through either rotations of rigid plates to yield structures such as tilted panels, rectangular cuboids, pyramids, or other hollow polyhedra, or rolling motions of flexible films to form tubes, scrolls, or other cylindrical shapes.

Self-folded structures with device-grade silicon provide new capabilities, such as higher efficiency and small form factor of capillary force-assisted 3D photovoltaic devices (Figure 3a). ${ }^{27}$ Here, the forces mediated by an evaporating water drop fold very thin $(1 \mu \mathrm{m})$, single-crystalline Si foils into deterministic shapes. The light-trapping that follows from these shapes improves the device performance by more than $300 \%$ compared to planar counterparts, and by more than $600 \%$ when combined with a $3 \times$ optical concentrator. ${ }^{27}$ Elsewhere, capillary forces have also been utilized to assemble a variety of off-planar and polyhedral geometries, including high Q microwave inductors, ${ }^{43}$ optomechanical structures, ${ }^{44}$ and polyhedra with device-grade silicon, ${ }^{25}$ 3D plasmonic resonators, ${ }^{45}$ Faraday cages, ${ }^{3}$ microcube antennas, and three-axis devices (Figure 3b-c). ${ }^{46}$

Newer approaches exploit the release of stress in prestrained elastomer substrates to cause 2D sheets of electronic materials or devices to spontaneously transform, without directed intervention, into 3D architectures with complex, targeted geometries (see Figure 3d). ${ }^{47,48}$ When patterned into appropriate geometries, functional membranes composed of advanced semiconductor materials can be delivered to prestretched elastomer substrates, where they chemically bond at a selected set of points, lithographically defined as spatial modulations in surface chemistry. Releasing the tension in the substrate induces compressive stresses that cause the unbound regions to lift out of the plane, with a trajectory in bending, twisting, and translating dictated by (1) the 2D layout of the original structure, (2) the configuration of bonding points, and (3) the magnitude and orientation of the prestretch. This simple process, which has some similarities to mechanisms in children's pop-up books, offers remarkable levels of versatility in 3D design, in a way that maintains compatibility with stateof-the-art 2D technologies, including those in electronics, photonics, and MEMS.

Demonstration experiments illustrate the ability to achieve more than 100 different 3D geometries using this technique, ranging from open-filamentary spirals, baskets, and networks, to chiral membranes, fractal plates, and closed-form architectures. ${ }^{47,48}$ In all cases, finite element analysis algorithms quantitatively capture the shapes, thereby providing indispensable design capabilities for rapidly exploring layout ideas. A unique feature of this mechanically directed approach to 3D assembly is that the structures can remain tethered to an underlying 


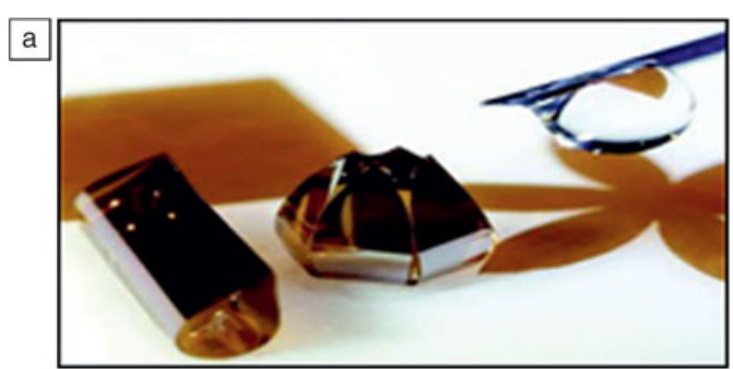

d

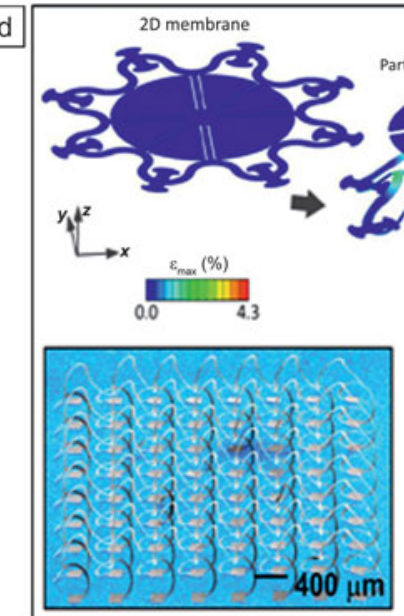

b
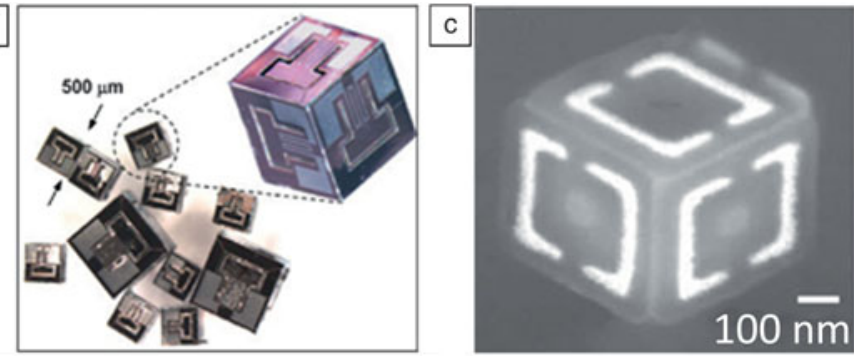

e

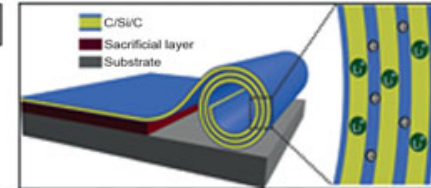

$\mathrm{f}$

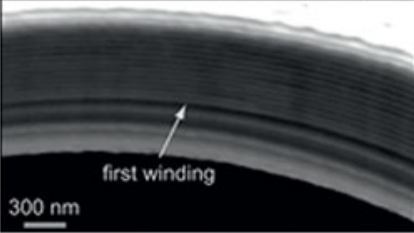

$\mathrm{g}$

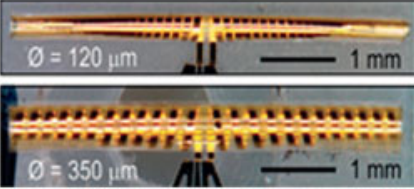

Figure 3. Electronic, electromagnetic, and energy devices. (a) Images of capillary-assisted folding of 3D, mm-scale photovoltaic devices. Reprinted with permission from Reference 27. Photograph courtesy of Xiaoying Guo, Zachery Johnson, and Alex Jerez (University of Illinois at Urbana-Champaign). (b) Optical images of capillary force-assembled and self-sealed magnetic field-sensitive three-axis sensors. ${ }^{46}$ (c) Scanning electron microscope image of a capillary-assisted self-folded cube with a 50-nm-thick, twin-loop, gold split-ring resonator defined by e-beam patterning on 50-nm-thick $\mathrm{Al}_{2} \mathrm{O}_{3}$ panels. Reprinted with permission from Reference 45. (c) 2011 Wiley. (d) Schematic and experimental images of pop-up origami using device-grade silicon on an elastomeric substrate (color scale shows maximum strain, $\varepsilon_{\max }$ ). Reprinted with permission from Reference 47. (C) 2015 American Association for the Advancement of Science. Reference 48. () 2015 National Academy of Sciences. (e) Schematic of self-rolled-up microtubes (S-RUMs) of $\mathrm{C} / \mathrm{Si} / \mathrm{C}$ and their use as anodes for lithium-ion batteries. Reprinted with permission from Reference 69. (C) 2013 Wiley. (f) Cross-sectional view of a S-RUM ultracompact capacitor comprising 13 windings and rolled from a 600- $\mu \mathrm{m}$-long planar capacitor. Reprinted with permission from Reference 54. (C) 2010 American Chemical Society. (g) Metallic S-RUM helical microantennas on a polymer bilayer with internal diameters $(\varnothing)$ indicated. Reprinted with permission from Reference 58. (C) 2015 Nature Publishing Group.

elastomer substrate, such that their 3D geometries can be reversibly adjusted through mechanical strain. Example devices include tunable spiral inductors, in which the 3D geometry, and therefore, the quality factor and resonance frequency, can be tuned, and rotating micromirrors whose tilt angles can be adjusted for beam steering and modulating the transmission.

Another 3D micro- and nanoscale construct of interest to materials used in electrical, electromagnetic, and energy devices is the self-rolled-up microtube (S-RUM). Here, strain induced by lattice, stoichiometric, or thermal mismatch deformation of ultrathin membranes made of semiconductors, dielectrics, metals, and polymers, or hybrid hierarchical composites, ${ }^{2,49-52}$ yield structures with cylindrical symmetry from planar precursors. ${ }^{53}$ Demonstrated devices include passive electronic components (capacitors, inductors, antennas, etc.) for radio frequency integrated circuit miniaturization, ${ }^{54-59}$ fully integrated field-effect transistors with ultrasmall bending radii, ${ }^{60}$ lasers, ${ }^{61,62}$ vertical ring resonators for $3 \mathrm{D}$ photonic integration, ${ }^{63-66}$ and batteries ${ }^{67-69}$ (Figure $3 \mathrm{e}-\mathrm{g}$ ). In terms of functionality, S-RUM inductors are 1-2 orders of magnitude smaller and $>5 \times$ higher in resonant frequency compared to their 2D counterparts (i.e., planar spiral inductors, with operation that is almost independent of substrate conductivity). ${ }^{55,56}$ Large-area S-RUM capacitor arrays and helical antennas can also be achieved. ${ }^{57,58}$ Pairs of inductors have been proposed as integrated microtransformers with near-perfect coupling coefficients. ${ }^{59,70}$ In photonics, S-RUMs naturally form ringresonator cavities. When coupled with III-V-based quantum dots or quantum wells as the gain materials, lasing is possible at ultralow thresholds, under both optical and electrical pumping. ${ }^{61,62} \mathrm{~S}-\mathrm{RUM}$ resonators provide convenient out-of-plane optical coupling schemes for 3D photonic integration, outside of the scope of possibilities with conventional planar microcavities. ${ }^{63-66}$ Evanescent fields along with the hollow core geometry make S-RUM resonators inherently suitable for optofluidic sensing and potentially for lab-on-a-chip applications. ${ }^{71,72}$ 


\section{Lithographically patterned metamaterials}

Metamaterials are synthetic and often micro- and nanostructured periodic or patterned structures that provide novel mechanical, electromagnetic, or chemical properties. Currently, a significant challenge is the parallel fabrication of true 3D metamaterials with material versatility and micro-/ nanoscale patterns. Origami approaches can be used to create building blocks such as micro- or nanopatterned polyhedra that can be tiled or aggregated to form 3D periodic metamaterials. ${ }^{73}$ Alternatively, micropatterned thin films can be folded up directly into complex materials with periodic 3D patterns, as demonstrated with metals, polymers, and hydrogels (Figure 4a-d) ${ }^{6,7,19,23,38,74,75}$ Previously assembled metamaterials include egg-crate-like, lithographically patterned self-folded sheets capable of supporting $128,000 \times$ their weight, negative Poisson ratio structures, and origami materials that facilitate mechanically stable lattice defects. ${ }^{23,75-77}$

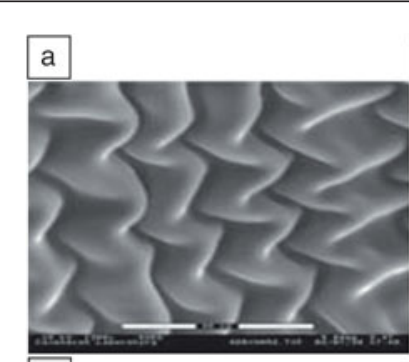

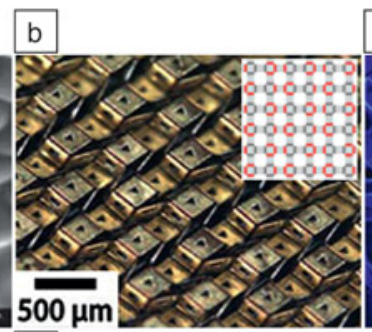

c
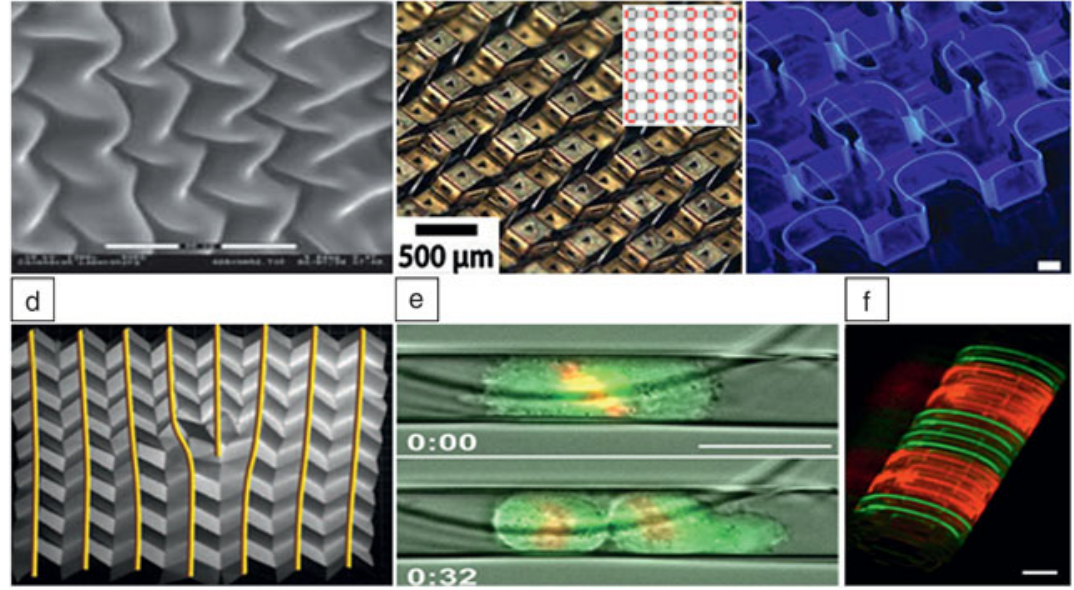

e

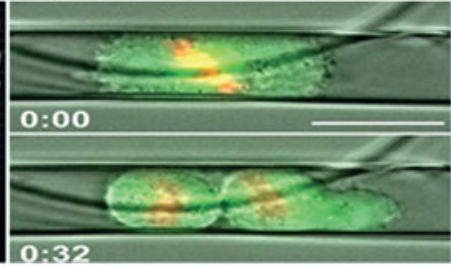

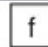

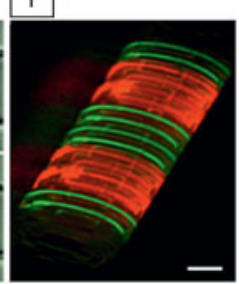

Figure 4. Metamaterials and tissue/cell engineering devices. (a) Zigzag Miura-ori patterns (a well-known rigid origami fold pattern) in a thin film atop a thick elastic substrate that is compressed biaxially, manifested here in a drying slab of gelatin with a thin skin that forms naturally and showing the physically driven self-organization of Miura-ori. Scale bar $35 \mu \mathrm{m}$. Reprinted with permission from Reference 74. () 2005 American Association for the Advancement of Science. (b) Metallic metamaterials with egg-crate-like patterns similar to that in Figure 1c, but self-folded and with significantly smaller dimensions; the materials have a 5:1 rigid segment to flexible hinge (45 $\mu \mathrm{m})$ ratio. Schematic shows the flat fold pattern with red lines representing $-90^{\circ}$ valley folds, dark lines representing $+90^{\circ}$ mountain folds, and gray areas representing rigid panels. Reprinted with permission from Reference 23. () 2009 American Institute of Physics. (c) Bidirectionally self-folded polymeric metamaterial with SU-8 (a photocrosslinkable epoxy) rigid faces and differentially cross-linked hinges. Scale bar $250 \mu \mathrm{m}$. Reprinted with permission from Reference 95. Image credits: Mustapha Jamal. () 2011 Nature Publishing Group. (d) Photograph of a paper sheet folded into a Miura-ori metamaterial with a complex defect structure, showing how columns of edge dislocations (highlighted yellow) generate a grain boundary. Reprinted with permission from Reference 75. (C) 2014 American Association for the Advancement of Science. (e) Fluorescent and phase-contrast time-lapse images of an entrapped, dividing HeLa cancer cell visualized with a green cytoskeleton imaged using GFP-tubulin and red nucleus imaged using the histone H2B-mCherry in a self-rolledup microtube device. Scale bar $15 \mu \mathrm{m}$. Reprinted with permission from Reference 86. (C) 2014 American Chemical Society. (f) Rolled-up polymeric device with dual fluidic channels through which flows fluorescein (green) or rhodamine B (red). Scale bar $500 \mu \mathrm{m}$. Reprinted with permission from Reference 95. Image credits: Mustapha Jamal. (c) 2011 Nature Publishing Group.
These demonstrations illustrate how complex curved and folded materials with hundreds to potentially millions of folds and patterns limited only by the resolution of planar lithog-

\section{Biomedical devices, robotics, and surgery}

A number of curved and folded structures have been assembled hat could mimic anatomical structures or confined geomevices (Figure 4e-f). For example, S-RUMs can guide and accelerate the outgrowth of neurons with exceptional directionality and speed, ${ }^{78,79}$ and control the mechanism of neuronal stem cell migration. ${ }^{80}$ Strain engineering has also been used to generate lab-in-a-tube devices, ${ }^{81}$ and curved and folded structures have been used to study the division of single cancer cells, microvascular mimics, drug delivery constructs, bioartificial organs and enable 3D microfluidic tissue scaffolds..$^{82-87}$

Out-of-plane bending, folding, twisting, and shape change can also be utilized to enable mechanical actuation of small 3D structures. Techniques such as microfabrication, printed circuit board (PCB) MEMS and lamination have been used to create structures that mimic insects or self-morphing robots (Figure 5a-d) ${ }^{88-90}$ At smaller size scales, untethered self-folding microgrippers have been used to biopsy gastrointestinal tissue in live pigs, deliver drugs, and capture single red blood cells (Figure 6a-c) ${ }^{91-93}$ S-RUM approaches have been used to create self-propelled structures such as catalytic micromotors that move by bubble propulsion (Figure 6d) and spermbots consisting of a S-RUM is attached to a sperm cell (Figure 6e). These devices have been used to perform a variety of tasks, such as movement of cargo, or even enable mechanized functions, such as drilling holes into cells and tissues. ${ }^{94}$ Challenges in robotic origami micro- and nanodevices include miniaturization, energy harvesting, speed, reversibility,materialversatility, biocompatibility, and programmability.

\section{Summary}

Techniques that utilize a range of micropatterning techniques such as photolithography, e-beam lithography, nanoimprint lithography, 3D printing, PCB MEMS, molding, and lamination can be combined with strain engineering to create a range of structures that can curve, fold, twist, or change their shape due to a variety of forces. As is evident from this article, a huge number of diverse structures and functionality have been enabled with a broad range of materials, including device-grade semiconductors, metals, inorganics, polymers, 

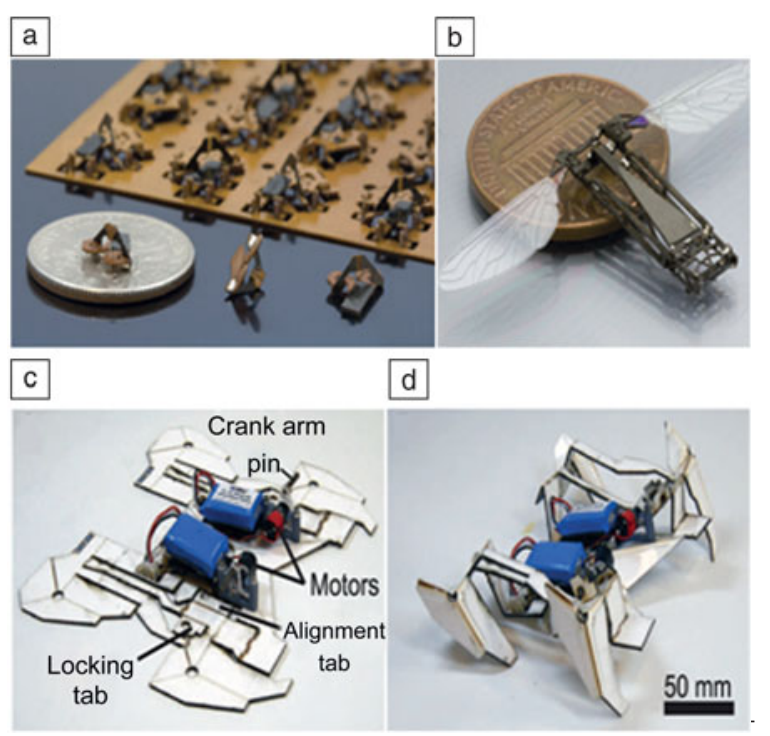

Figure 5. Robots. (a) Spherical five-bar mechanical linkages fabricated from printed circuit board (PCB)microelectromechanical (MEMS), in front of a larger panel used to mass-produce them. A US dime provides scale. (b) A monolithic bee (Mobee) fabricated using PCB-MEMS (a US penny provides scale). (a-b) Reprinted with permission from Reference 89. (C) 2012 IOP Publishing. (c-d) Self-assembly steps of a cm-scale robot by folding. Reprinted with permission from Reference 96. (C) 2014 American Association for the Advancement of Science.

and hydrogels. Future challenges are to further broaden the material versatility, especially with high-quality semiconductors, natural biomaterials, living cells, and 2D layered materials, which will broaden applicability.

There is still much that needs to be understood on the theoretical side in terms of mechanics modeling and programmability; for example, the inclusion of biologically inspired concepts such as hierarchy of folds, orthogonality of interactions, and multistate assembly could enable complex functionality. In addition, the focus in the next decade should include issues such as manufacturability, yield, and reliability, as these processes move from the laboratory and into practical real-world applications.

\section{Acknowledgments}

D.H.G. acknowledges support from the National Science Foundation (CMMI 1200241 and DMR-1507749). Y.H. acknowledges support from the National Science Foundation (CMMI1400169) and from the US National Institutes of Health (NIMAS R01 AR051376 and R01 AR058004). We thank Seung Hyun Oh and ChangKyu Yoon for help with preparation of the figures.

\section{References}

1. R.R.A. Syms, E.M. Yeatman, V.M. Bright, G.M. Whitesides, J. Microelectromech. Syst. 12, 387 (2003).

2. X. Li, J. Phys. D Appl. Phys. 41, 193001 (2008).

3. T.G. Leong, A.M. Zarafshar, D.H. Gracias, Small 6, 792 (2010).

4. J.S. Randhawa, K.E. Laflin, N. Seelam, D.H. Gracias, Adv. Funct. Mater. 21, 2395 (2011).
5. Y.F. Mei, A.A. Solovev, S. Sanchez, O.G. Schmidt, Chem. Soc. Rev. 40 , 2109 (2011).

6. V.B. Shenoy, D.H. Gracias, Mater. Res. Bull. 13, 847 (2012).

7. D.H. Gracias, Curr. Opin. Chem. Eng. 2, 112 (2013).

8. E.A. Peraza-Hernandez, D.J. Hartl, R.J. Malak, D.C. Lagoudas, Smart Mater. Struct. 23, 094001 (2014).

9. F. Cavallo, M.G. Lagally, Nano Today 10, 538 (2015).

10. K.S.J. Pister, M.W. Judy, S.R. Burgett, R.S. Fearing, Sens. Actuators A $\mathbf{3 3}$, 249 (1992).

11. E.E. Hui, R.T. Howe, M.S. Rodgers, Proc. Thirteenth Annu. Int. Conf. on Microelectromech. Syst. (2000), p. 602.

12. S.T. Brittain, O.J.A. Schueller, H.K. Wu, S. Whitesides, G.M. Whitesides, J. Phys. Chem. B 105, 347 (2001).

13. T. Nojima, K. Saito, JSME Int. J. Ser. A 49, 38 (2006).

14. I. Stewart, Nature 448, 419 (2007).

15. B.Y. Ahn, D. Shoji, C.J. Hansen, E. Hong, D.C. Dunand, J.A. Lewis, Adv. Mater. 22, 2251 (2010).

16. H.J. In, S. Kumar, Y. Shao-Horn, G. Barbastathis, Appl. Phys. Lett. 88, $083104(2006)$

17. R.H. Baughman, L.W. Shacklette, R.L. Elsenbaumer, E.J. Plichta, C. Becht, in Molecular Electronics, P.I. Lazarev, Ed. (Springer Netherlands, Dordrecht, 1991), p. 267. 18. E. Smela, O. Inganas, I. Lundstrom, Science 268, 1735 (1995).

19. E. Hawkes, B. An, N.M. Benbernou, H. Tanaka, S. Kim, E.D. Demaine, D. Rus, R.J. Wood, Proc. Natl. Acad. Sci. U.S.A. 107, 12441 (2010).

20. V.Y. Prinz, D. Grutzmacher, A. Beyer, C. David, B. Ketterer, E. Deckardt, Nanotechnology 12, 399 (2001).

21. O.G. Schmidt, C. Deneke, Y.M. Manz, C. Muller, Physica E13, 969 (2002).

22. P.O. Vaccaro, K. Kubota, T. Fleischmann, S. Saravanan, T. Aida, Microelectron. J. 34, 447 (2003).

23. N. Bassik, G.M. Stern, D.H. Gracias, Appl. Phys. Lett. 95, 091901 (2009).

24. P.W. Green, R.R.A. Syms, E.M. Yeatman, J. Microelectromech. Syst. 4, 170 (1995).

25. D.H. Gracias, V. Kavthekar, J.C. Love, K.E. Paul, G.M. Whitesides, Adv. Mater. 14, 235 (2002).

26. C. Py, P. Reverdy, L. Doppler, J. Bico, B. Roman, C.N. Baroud, Phys. Rev. Lett. 98, 156103 (2007).

27. X.Y. Guo, H. Li, B.Y. Ahn, E.B. Duoss, K.J. Hsia, J.A. Lewis, R.G. Nuzzo, Proc. Natl. Acad. Sci. U.S.A. 106, 20149 (2009).

28. J.W. Judy, R.S. Muller, J. Microelectromech. Syst. 6, 249 (1997).

29. Y.W. Yi, C. Liu, J. Microelectromech. Syst. 8, 10 (1999).

30. Z.B. Hu, X.M. Zhang, Y. Li, Science 269, 525 (1995).

31. Y. Klein, E. Efrati, E. Sharon, Science 315, 1116 (2007)

32. L. Ionov, Soft Matter 7, 6786 (2011).

33. J. Kim, J.A. Hanna, M. Byun, C.D. Santangelo, R.C. Hayward, Science $\mathbf{3 3 5}$, 1201 (2012)

34. Y. Liu, J.K. Boyles, J. Genzer, M.D. Dickey, Soft Matter 8, 1764 (2012).

35. D.Y. Chen, J. Yoon, D. Chandra, A.J. Crosby, R.C. Hayward, J. Polym. Sci. B Polym. Phys. 52, 1441 (2014).

36. T.H. Ware, M.E. McConney, J.J. Wie, V.P. Tondiglia, T.J. White, Science $\mathbf{3 4 7}$, $982(2015)$

37. S. Pandey, M. Ewing, A. Kunas, N. Nguyen, D.H. Gracias, G. Menon, Proc. Natl. Acad. Sci. U.S.A. 108, 19885 (2011).

38. R. Nagpal, Proc. 1st Intl. Joint Conf. on Autonomous Agents and Multiagent Syst. Part 1 (ACM 2002), p. 418.

39. N.M. Benbernou, E.D. Demaine, M.L. Demaine, A. Ovadya, in Origami 5: Fifth International Meeting of Origami Science, Mathematics, and Education, P. Wang-Iverson, R.J. Lang, M. Yim, Eds. (A K Peters/CRC Press, Boca Raton, FL, 2011), p. 405.

40. M.T. Tolley, S.M. Felton, S. Miyashita, D. Aukes, D. Rus, R.J. Wood, Smart Mater. Struct. 23094006 (2014).

41. B. An, D. Rus, Rob. Auton. Syst. 62, 976 (2014).

42. D. Raviv, W. Zhao, C. McKnelly, A. Papadopoulou, A. Kadambi, B.X. Shi, S. Hirsch, D. Dikovsky, M. Zyracki, C. Olguin, R. Raskar, S. Tibbits, Sci. Rep. 4, 7422 (2014).

43. G.W. Dahlmann, E.M. Yeatman, Electron. Lett. 36, 1707 (2000).

44. R.R.A. Syms, J. Microelectromech. Syst. 8, 448 (1999).

45. J.H. Cho, M.D. Keung, N. Verellen, L. Lagae, V.V. Moshchalkov, P. Van Dorpe, D.H. Gracias, Small 7, 1943 (2011).

46. D. Gracias, J.-H. Cho, S. Hu, in Mater. Res. Soc. Symp. Proc. 1249, C. Bonafos, Y. Fujisaki, P. Dimitrakis, E. Tokumitsu, Eds. (Materials Research Society, Warrendale, PA, 2010), p. F09-07.

47. S. Xu, Z. Yan, K.I. Jang, W. Huang, H.R. Fu, J. Kim, Z. Wei, M. Flavin, J. McCracken, R. Wang, A. Badea, Y. Liu, D.Q. Xiao, G.Y. Zhou, J. Lee, H.U. Chung, H.Y. Cheng, W. Ren, A. Banks, X.L. Li, U. Paik, R.G. Nuzzo, Y.G. Huang, Y.H. Zhang, J.A. Rogers, Science 347, 154 (2015).

48. Y. Zhang, Z. Yan, K. Nan, D. Xiao, Y. Liu, H. Luan, H. Fu, X. Wang, Q. Yang, J. Wang, W. Ren, H. Si, F. Liu, L. Yang, H. Li, J. Wang, X. Guo, H. Luo, L. Wang, Y. Huang, J.A. Rogers, Proc. Natl. Acad. Sci. U.S.A. 112, 11757 (2015).

49. V. Ya. Prinz, V.A. Seleznev, A.K. Gutakovsky, A.V. Chehovskiy, V.V. Preobrazhenskii, M.A. Putyato, T.A. Gavrilova, Physica E 6, 828 (2000). 


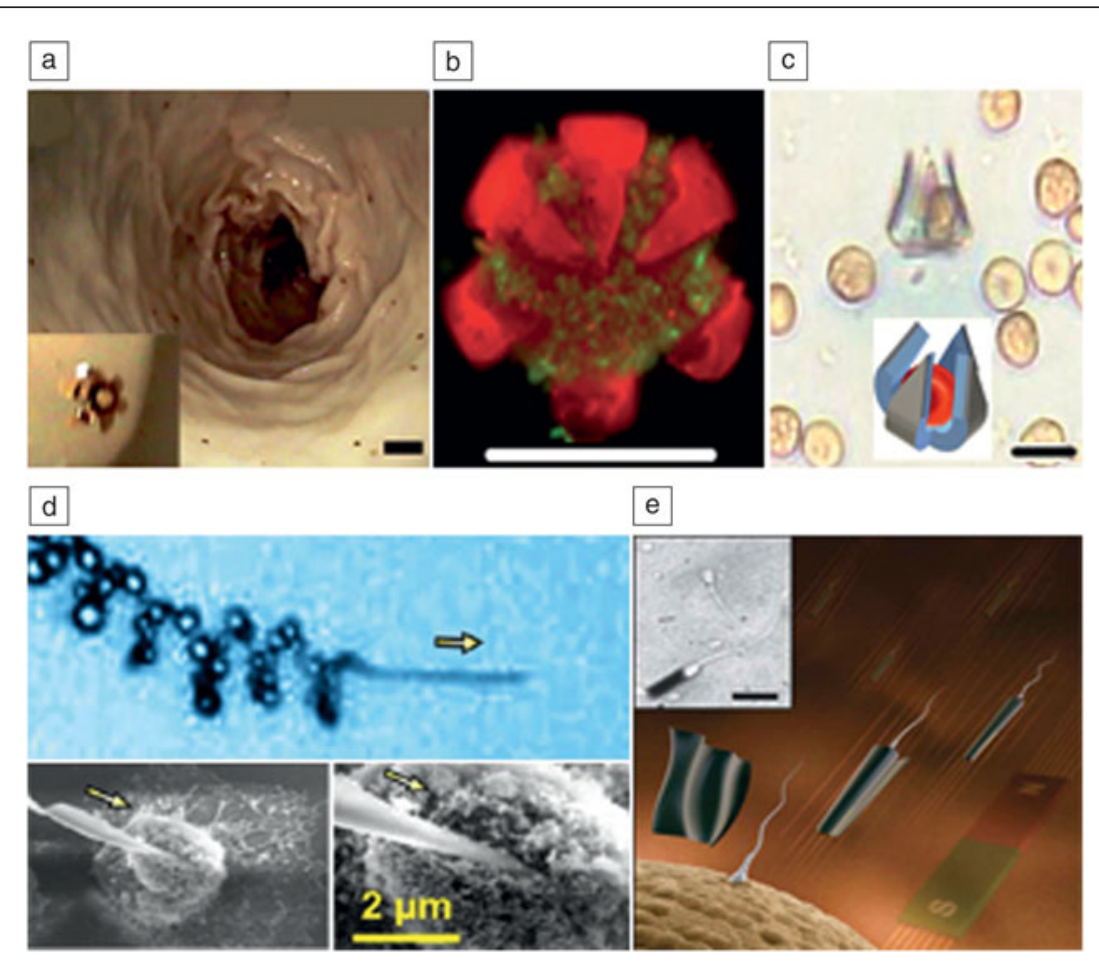

Figure 6. Micro- and nanosurgical tools. (a) Endoscopic and zoomed image of dust-sized, self-folding microgrippers inside a porcine colon. Scale bar $2 \mathrm{~mm}$. Inset shows zoom of the microgrippers (black spots in the larger image). The size of the gripper shown in the inset is $1 \mathrm{~mm}$ when fully open. Reprinted with permission from Reference 91. (C) 2013 Elsevier. (b) Optical and fluorescent images of all-polymeric, self-folding theragrippers tightly closed around and gripping a clump of cells. Scale bar is $1 \mathrm{~mm}$. Reprinted with permission from Reference 92. () 2014 Wiley. (c) Optical image and schematic of self-folding single-cell grippers holding onto a red blood cell. Scale bar $10 \mu \mathrm{m}$. Reprinted with permission from Reference 93. (C) 2014 American Chemical Society. (d) Tapered catalytic self-rolled-up microtube (S-RUM) catalytic self-propelled motors moving in a screw-like motion (top image) and drilling into a single fixed HeLa cancer cell (bottom images). Reprinted with permission from Reference 97. (C) 2011 American Chemical Society. (e) The vision of spermbots: capture and delivery of single spermatozoa to the oocyte (egg cell) by a magnetic microtube that is controlled by an external magnetic field. The inset shows a microscopic image of a bovine spermatozoon entering a $20-\mu \mathrm{m}$-long S-RUM. Scale bar $20 \mu \mathrm{m}$. Reprinted with permission from Reference 94. (C) 2014 Informa UK, Ltd.

62. M.H.T. Dastjerdi, M. Djavid, Z. Mi, Appl. Phys. Lett. 106, 021114 (2015).

63. Z.B. Tian, V. Veerasubramanian, P. Bianucci, S. Mukherjee, Z.T. Mi, A.G. Kirk, D.V. Plant, Opt. Express 19, 12164 (2011). 64. S. Böttner, S. Li, M.R. Jorgensen, O.G. Schmidt, Appl. Phys. Lett. 102, 251119 (2013).

65. X. Yu, E. Arbabi, L.L. Goddard, X. Li, X. Chen, Appl. Phys. Lett. 107, 031102 (2015).

66. A. Madani, M. Kleinert, D. Stolarek, L. Zimmermann, L. Ma, O.G. Schmidt, Opt. Lett. 40, 3826 (2015).

67. C.L. Yan, W. Xi, W.P. Si, J.W. Deng, O.G. Schmidt, Adv. Mater. 25, 539 (2013).

68. W.P. Si, I. Monch, C.L. Yan, J.W. Deng, S.L. Li, G.G. Lin, L.Y. Han, Y.F. Mei, O.G. Schmidt, Adv. Mater. 26, 7973 (2014). 69. J.W. Deng, H.X. Ji, C.L. Yan, J.X. Zhang, W.P. Si, S. Baunack, S. Oswald, Y.F. Mei, O.G. Schmidt, Angew. Chem. Int. Ed. 52, 2326 (2013).

70. 0.G. Schmidt, C. Deneke, S. Kiravittaya, R. Songmuang, H. Heidemeyer, Y. Nakamura, R. Zapf-Gottwick, C. Muller, N.Y. Jin-Phillipp, IEEE J. Sel. Top. Quantum Electron. 8, 1025 (2002). 71. A. Bernardi, S. Kiravittaya, A. Rastelli, R. Songmuang, D. Thurmer, M. Benyoucef, O.G. Schmidt, Appl. Phys. Lett. 93, 094106 (2008)

72. S.M. Harazim, V.A.B. Quiñones, S. Kiravittaya, S. Sanchez, 0.G. Schmidt, Lab Chip 12, 2649 (2012).

73. J.S. Randhawa, S.S. Gurbani, M.D. Keung, D.P. Demers, M.R. Leahy-Hoppa, D.H. Gracias, Appl. Phys. Lett. 96, 191108 (2010).

74. L. Mahadevan, S. Rica, Science 307, 1740 (2005).

75. J.L. Silverberg, A.A. Evans, L. McLeod, R.C. Hayward, T. Hull, C.D. Santangelo, I. Cohen, Science 345, 647 (2014). 76. H. Yasuda, J. Yang, Phys. Rev. Lett. 114, 185502 (2015). 77. J.L. Silverberg, J.H. Na, A.A. Evans, B. Liu, T.C. Hull, C.D. Santangelo, R.J. Lang, R.C. Hayward, I. Cohen, Nat. Mater. 14, 540 (2015).

78. S. Schulze, G.S. Huang, M. Krause, D. Aubyn, V.A.B. Quinones, C.K. Schmidt, Y.F. Mei, O.G. Schmidt, Adv. Eng. Mater. 12, B558 (2010)

79. P. Froeter, Y. Huang, O.V. Cangellaris, W. Huang, E.W. Dent, M.U. Gillette, J.C. Williams, X. L. Li, ACS Nano 8, 11108 (2014). 80. B. Koch, A.K. Meyer, L. Helbig, S. Harazim, A. Storch, S. Sanchez, O.G. Schmidt, Nano Lett. 15, 5530 (2015).

81. E.J. Smith, W. Xi, D. Makarov, I. Mönch, S. Harazim, V.A.B. Quiñones, C.K. Schmidt, Y. Mei, S. Sanchez, O.G. Schmidt, Lab Chip 12, 1917 (2012).

82. C.L. Randall, E. Gultepe, D.H. Gracias, Trends Biotech. 30, 138 (2012).

83. E.J. Smith, S. Schulze, S. Kiravittaya, Y.F. Mei, S. Sanchez, 0.G. Schmidt, Nano Lett. 11, 4037 (2011).

84. R. Fernandes, D.H. Gracias, Adv. Drug Deliv. Rev. 64, 1579 (2012).

85. V. Stroganov, S. Zakharchenko, E. Sperling, A.K. Meyer, O.G. Schmidt, L. Ionov, Adv. Funct. Mater. 24, 4357 (2014).

50. O.G. Schmidt, K. Eberl, Nature 410, 168 (2001)

51. Y.F. Mei, G.S. Huang, A.A. Solovev, E.B. Urena, I. Moench, F. Ding, T. Reindl, R.K.Y. Fu, P.K. Chu, O.G. Schmidt, Adv. Mater. 20, 4085 (2008).

52. Z. Mi, P. Bianucci, Curr. Opin. Solid State Mater. Sci. 16, 52 (2012).

53. W. Huang, S. Koric, X. Yu, K.J. Hsia, X.L. Li, Nano Lett. 14, 6293 (2014).

54. C.C.B. Bufon, J.D.C. Gonzalez, D.J. Thurmer, D. Grimm, M. Bauer, O.G. Schmidt, Nano Lett. 10, 2506 (2010).

55. W. Huang, X. Yu, P. Froeter, R.M. Xu, P. Ferreira, X.L. Li, Nano Lett. 12, $6283(2012)$

56. P. Froeter, X. Yu, W. Huang, F. Du, M.Y. Li, I. Chun, S.H. Kim, K.J. Hsia, J.A. Rogers, X.L. Li, Nanotechnology 24, 475301 (2013).

57. R. Sharma, C.C.B. Bufon, D. Grimm, R. Sommer, A. Wollatz, J. Schadewald, D.J. Thurmer, P.F. Siles, M. Bauer, O.G. Schmidt, Adv. Energy Mater. 4, 1301631 (2014).

58. D.D. Karnaushenko, D. Karnaushenko, D. Makarov, O.G. Schmidt, NPG Asia Mater. 7, e188 (2015).

59. W. Huang, M. Li, S. Gong, X. Li, "Self-rolled-up Tube Transformers: Extreme Miniaturization and Performance Enhancement," presented at the 73rd Annual Device Research Conference (IEEE Electron Devices Society), The Ohio State University, Columbus, OH, June 21-24, 2015, p. 223.

60. D. Grimm, C.C.B. Bufon, C. Deneke, P. Atkinson, D.J. Thurmer, F. Schaffel, S. Gorantla, A. Bachmatiuk, O.G. Schmidt, Nano Lett. 13, 213 (2013).

61. P. Bianucci, S. Mukherjee, M.H.T. Dastjerdi, P.J. Poole, Z. Mi, Appl. Phys. Lett. 101, 031104 (2012).
86. W. Xi, C.K. Schmidt, S. Sanchez, D.H. Gracias, R.E. Carazo-Salas, S.P. Jackson, O.G. Schmidt, Nano Lett. 14, 4197 (2014).

87. R. Arayanarakool, A.K. Meyer, L. Helbig, S. Sanchez, O.G. Schmidt, Lab Chip 15, 2981 (2015).

88. K. Suzuki, I. Shimoyama, H. Miura, Y. Ezura, "Creation of an Insect-based Microrobot with an External Skeleton and Elastic Joints," Proc. IEEE Microelectromech. Syst. (MEMS '92), (1992), p. 190.

89. P.S. Sreetharan, J.P. Whitney, M.D. Strauss, R.J. Wood, J. Micromech. Microeng. 22055027 (2012).

90. J. Paik, B. An, D. Rus, R.J. Wood, Proc. 2nd Int. Conf. on Morphological Computation (ICMC 2011), (2012), p. 12

91. E. Gultepe, S. Yamanaka, K.E. Laflin, S. Kadam, Y. Shim, A.V. Olaru, B. Limketkai, M.A. Khashab, A.N. Kalloo, D.H. Gracias, F.M. Selaru, Gastroenterology 144, 691 (2013).

92. K. Malachowski, J. Breger, H.R. Kwag, M.O. Wang, J.P. Fisher, F.M. Selaru, D.H. Gracias, Angew. Chem. Int. Ed. 53, 8045 (2014).

93. K. Malachowski, M. Jamal, Q.R. Jin, B. Polat, C.J. Morris, D.H. Gracias, Nano Lett. 14, 4164 (2014).

94. V. Magdanz, M. Guix, O.G. Schmidt, Robotics Biomim. 1, 1 (2014).

95. M. Jamal, A.M. Zarafshar, D.H. Gracias, Nat. Commun. 2, 527 (2011).

96. S. Felton, M. Tolley, E. Demaine, D. Rus, R. Wood, Science 345, 644 (2014). 97. A.A. Solovev, W. Xi, D.H. Gracias, S.M. Harazim, C. Deneke, S. Sanchez, 0.G. Schmidt, ACS Nano 6, 1751 (2012). 